\title{
Fragmentation Arising from a Distributional Initial Condition II
}

\author{
G.C. McGuinness, W. Lamb*, A.C. McBride, \\ Department of Mathematics and Statistics, University of Strathclyde, \\ Livingstone Tower, 26 Richmond Street, Glasgow G1 1XH, Scotland, U.K.
}

November 15, 2010

\begin{abstract}
We consider a class of fragmentation equations in which the distribution of daughter particles formed when a parent particle fragments is governed by a homogeneous function. A systematic procedure is presented for constructing a space of distributions in which initial-value problems involving singular initial conditions can be analysed. This procedure makes use of results on sun dual semigroups and equicontinuous semigroups on locally convex spaces. Explicit solutions are obtained for the the case when the fragmentation processes are governed by power-law kernels and have monodisperse initial conditions modelled by Dirac delta distributions. Rigorous justification is thereby provided for results obtained more formally by Ziff and McGrady.
\end{abstract}

AMS subject classifications: 47D06, 34G10, 45K05, 46F05.

Abbreviated Title: Distributional Fragmentation.

Key words. Fragmentation, Abstract Cauchy Problem, Equicontinuous Semigroup, Dirac Delta.

\section{Introduction}

In this paper we continue our investigations into the initial-value problem

$$
\begin{aligned}
\frac{\partial}{\partial t} u(x, t) & =-a(x) u(x, t)+\int_{x}^{\infty} r\left(\frac{x}{y}\right) a(y) u(y, t) \frac{d y}{y} \\
u(x, 0) & =u_{0}(x) .
\end{aligned}
$$

Problems of this type arise as mathematical models of fragmentation processes in which $u(x, t)$ is interpreted as the density of particles of mass $x$ at time $t$. The terms on the right-hand side of (1.1)

\footnotetext{
${ }^{*}$ Corresponding author. Fax: +44(00) 1415483345

E-mail addresses / Telephone numbers:

w.lamb@strath.ac.uk, +44(00) 1415483649 (W. Lamb);

a.c.mcbride@strath.ac.uk, +44(00) 1415483647 (A.C. McBride).
} 
represent, respectively, the loss of particles of mass $x$ due to their break-up into smaller particles and the gain in particles of mass $x$ due to the fragmentation of particles of masses $y>x$. The distribution of particles of mass $x$ produced when a particle of mass $y$ fragments is governed by the function $r(x / y) / y$. This "homogeneous" form corresponds to the assumption that the distribution of daughter particles is determined by the ratio daughter mass/parent mass.

We are interested in the case when the initial condition (1.2) involves a distribution, such as $u_{0}(x)=\delta(x-\ell)$ for some $\ell>0$, where $\delta$ is the Dirac delta. In fragmentation processes, monodisperse initial conditions are modelled using delta functionals of this form, and some explicit solutions of the resulting initial-value problems have been obtained for particular functions $r$ by Ziff and McGrady in [1]-[3]. As the approach used by Ziff and McGrady is based on formal arguments in which the Dirac delta is treated as a classical function, our primary objective here is to develop a mathematically rigorous method that can be used effectively in the analysis of fragmentation equations involving non-classical initial data.

Following [4, Chapter 8], we aim to recast (1.1)-(1.2) as an abstract Cauchy problem (ACP) of the form

$$
\frac{d}{d t} u(t)=K u(t) \quad(t>0) ; \quad u(0)=u_{0}
$$

where $K$ is an appropriately-defined operator realisation of the mapping

$$
\phi(x) \rightarrow-a(x) \phi(x)+\int_{x}^{\infty} r\left(\frac{x}{y}\right) a(y) \phi(y) \frac{d y}{y}, x>0 .
$$

However, instead of the usual setting of the Banach space $L_{1}\left(\mathbb{R}_{+}, x d x\right)$ that is adopted in [4], the ACP will be studied in suitable spaces of test-functions and their duals.

Of particular interest is the case when the functions $a$ and $r$ appearing in (1.1) have the form

$$
a(x)=x^{\alpha+1}, \quad r(x)=(\nu+2) x^{\nu}(x>0)
$$

where $\alpha$ and $\nu$ are real constants. This power law case has been studied by Ziff and McGrady [1]-[3] and is also discussed in [4, Chapter 8]. In [5] we examined the special case $\alpha=-1$ for which the corresponding operator $K$ in (1.3) (denoted by $A$ in [5]) is bounded in the space $L_{1}\left(\mathbb{R}_{+}, x d x\right)$. However, when $\alpha>-1$, we lose boundedness and the theory becomes more complicated. Difficulties arise because the adjoint semigroup of the $C_{0}$-semigroup $\left\{S_{K}(t)\right\}_{t \geq 0}$ generated by $K$ need not be strongly continuous. One way to overcome this is to use the theory of sun duals (equivalently known as semigroup duals) and sun dual semigroups and this is the approach we shall adopt below. In Section 2 we present some theory related to the (non-reflexive) space $L_{1}\left(\mathbb{R}_{+}, x d x\right)$ which we shall require in the sequel. Sections 3 and 4 are concerned with relevant spaces of test-functions 
and the corresponding generalised functions, respectively. Finally, in Section 5, we establish the main results concerning the appropriate distributional version of the ACP (1.3) when $a$ and $r$ are given by (1.4).

\section{Existence and Uniqueness Results in $L_{1}\left(\mathbb{R}_{+}, x d x\right)$}

As in [5], we let

$$
X=L_{1}\left(\mathbb{R}_{+}, x d x\right):=\left\{\phi:\|\phi\|_{X}:=\int_{0}^{\infty} x|\phi(x)| d x<\infty\right\}
$$

Adopting the notation used in [4], we introduce operators $A$ and $B$ defined in $X$ by

$$
\begin{aligned}
& (A \phi)(x):=-a(x) \phi(x), \quad D(A):=\{\phi \in X: a \phi \in X\} \\
& (B \phi)(x):=\int_{x}^{\infty} r\left(\frac{x}{y}\right) a(y) \phi(y) \frac{d y}{y}, \quad D(B):=D(A) .
\end{aligned}
$$

The non-negative functions $a$ and $r$, defined on $\mathbb{R}_{+}$, are required to satisfy the following conditions:

$$
\text { (C.1) } \quad \limsup _{x \rightarrow 0+} a(x)<\infty ; \quad \text { (C.2) } \quad \int_{0}^{1} x r(x) d x=1 .
$$

In what follows, it is convenient to express the operator $B$ as the composition $-R A$, where

$$
(R \phi)(x):=\int_{x}^{\infty} r\left(\frac{x}{y}\right) \phi(y) \frac{d y}{y}, \quad \phi \in X
$$

Condition (C.2) ensures that $R \in B(X)$ with $\|R\|_{B(X)}=1$.

Theorem 2.1 Let $K=\overline{A+B}$, where $\overline{A+B}$ denotes the closure of the operator $(A+B, D(A))$. Then $K$ is the infinitesimal generator of a positive semigroup $\left\{S_{K}(t)\right\}_{t \geq 0}$ of isometries on $X$. Therefore the ACP

$$
\frac{d}{d t} u(t)=K u(t)(t>0) ; \quad u(0)=u_{0},
$$

has a unique non-negative, strongly continuously differentiable solution $u:[0, \infty) \rightarrow D(K)$ for all non-negative $u_{0} \in D(K)$, given by

$$
u(t)=S_{K}(t) u_{0}, \quad t \geq 0 .
$$

Moreover $\|u(t)\|_{X}=\left\|u_{0}\right\|_{X}$ for all $t \geq 0$.

Proof See [4, Theorems 8.3 and 8.5]. 
In the case when the function $a$ is given by $a(x)=x^{\alpha+1}$, where $\alpha \geq-1$, it is also possible to establish an analogous result for the ACP

$$
\frac{d}{d t} u(t)=A u(t)+B u(t)(t>0) ; \quad u(0)=u_{0} \in D(A) .
$$

Once again, the unique solution is given by (2.6), as it can be shown that $D(A)$ is invariant under $S_{K}(t)$ for all $t>0$. Details can be found in [6, Example 6.4].

We recall from [5] that the dual space of $X$ can be identified with the Banach space $Y$ given by

$$
Y:=\left\{\psi: \psi \text { is measurable on } \mathbb{R}_{+} \text {and }\|\psi\|_{Y}<\infty\right\}
$$

where

$$
\|\psi\|_{Y}=\operatorname{ess} \sup _{x \in \mathbb{R}_{+}} \frac{1}{x}|\psi(x)|=\left\|\frac{1}{x} \psi(x)\right\|_{\infty},
$$

and the duality pairing is given by

$$
(\psi, \phi)=\int_{0}^{\infty} \psi(x) \phi(x) d x \quad(\psi \in Y, \phi \in X) .
$$

Each operator $S_{K}(t)$ in $(2.6)$ has a unique adjoint $S_{K}^{*}(t)$ on $Y$ with respect to the duality $(2.10)$, satisfying

$$
\left(S_{K}^{*}(t) \psi, \phi\right)=\left(\psi, S_{K}(t) \phi\right) \quad(\psi \in Y, \phi \in X, t \geq 0) .
$$

It is straightforward to check that $\left\{S_{K}^{*}(t)\right\}_{t \geq 0}$ is a semigroup on $Y$. However, since $X$ is not a reflexive Banach space, we cannot deduce that this adjoint semigroup is strongly continuous. To overcome this problem, we shall invoke the theory of sun duals; see [7, Section 2.6].

\section{Definition 2.2}

(i) The sun dual of $X$ with respect to $K$ is the set

$$
X^{\odot}(K):=\left\{\psi \in Y: S_{K}^{*}(t) \psi \rightarrow \psi \text { in } Y \text { as } t \rightarrow 0^{+}\right\}
$$

(ii) For each $t \geq 0$, the operator $S_{K}^{\odot}(t)$ is defined to be the restriction of $S_{K}^{*}(t)$ to the $\left(S_{K}^{*}(t)\right.$ invariant) subspace $X^{\odot}(K)$.

Theorem 2.3 The family $\left\{S_{K}^{\odot}(t)\right\}_{t \geq 0}$ forms a contraction semigroup on the Banach space $X^{\odot}(K)$. Moreover,

$$
X^{\odot}(K)=\overline{D\left(K^{*}\right)} .
$$


Proof From [7, Section 2.6], $X^{\odot}(K)$ is a Banach space, $(2.13)$ holds and $\left\{S_{K}^{\odot}(t)\right\}_{t \geq 0}$ is a strongly continuous semigroup. Finally, since $S_{K}(t)$ is an isometry,

$$
\left\|S_{K}^{\odot}(t)\right\|_{X \odot(K)} \leq\left\|S_{K}^{*}(t)\right\|_{X^{*}}=\left\|S_{K}(t)\right\|_{X}=1
$$

Let $K^{\odot}$ denote the infinitesimal generator of $\left\{S_{K}^{\odot}(t)\right\}_{t \geq 0}$. Then, by [7, p.63], $K^{\odot}$ is the part of $K^{*}$ in $X^{\odot}(K)$. Now consider the operator $A+B$ defined on $D(A+B):=D(A)$ via (2.2) and (2.3). By $\left[7\right.$, p.65], the operator $A$ is the infinitesimal generator of a $C_{0}$-semigroup on $X$, and therefore

$$
\overline{D(A+B)}=\overline{D(A)}=X
$$

Consequently, $(A+B)^{*}$ is a well-defined operator in $Y$. Moreover, as $K=\overline{(A+B)}$ (by Theorem 2.1), it follows that $K^{*}=(A+B)^{*}$ and so $K^{\odot}=(A+B)^{\odot}$, where the latter is the part of $(A+B)^{*}$ in $X^{\odot}(K)$; i.e.

$$
D\left(K^{\odot}\right)=D\left((A+B)^{\odot}\right)=\left\{\psi \in D\left((A+B)^{*}\right):(A+B)^{*} \psi \in X^{\odot}(K)\right\}
$$

and

$$
K^{\odot} \psi=(A+B)^{\odot} \psi=(A+B)^{*} \psi, \quad \psi \in D\left(K^{\odot}\right) .
$$

The following result provides a useful representation of $(A+B)^{*}$.

Theorem 2.4 As operators on $Y$,

$$
(A+B)^{*}=A^{*}\left(I-R^{*}\right)
$$

where $R^{*}$ is the adjoint of the operator $R \in B(X)$ given by (2.4).

Proof Let $\phi \in D(K)$ and $\psi \in D\left((A+B)^{*}\right)$. Then

$$
\left.(\psi, K \phi)=\left((A+B)^{*} \psi, \phi\right)=((I-R) A)^{*} \psi, \phi\right) .
$$

Since $(I-R) \in B(X)$, standard results on adjoints lead to

$$
(A+B)^{*}=[(I-R) A]^{*}=A^{*}(I-R)^{*}=A^{*}\left(I-R^{*}\right) .
$$

Corollary 2.5 The semigroup dual of $X$ with respect to $S_{K}(t)$ satisfies the equation

$$
X^{\odot}(K)=\overline{D\left(A^{*}\left(I-R^{*}\right)\right)} .
$$


Proof As $K^{*}=(A+B)^{*}$, the result follows by using (2.15) in (2.13).

Theorem 2.6 The ACP

$$
\frac{d}{d t} u(t)=(A+B)^{*} u(t)(t>0) ; \quad u(0)=u_{0}
$$

has a unique strongly continuously differentiable solution for all $u_{0} \in D\left(K^{\odot}\right)$ given by

$$
u(t)=S_{K}^{\odot}(t) u_{0}(t \geq 0)
$$

Proof By Theorem 2.3, we know that the family of operators $\left\{S_{K}^{\odot}(t)\right\}_{t \geq 0}$ forms a contractive $C_{0}$-semigroup on $X^{\odot}(K)$. Further, $K^{\odot}$ is the infinitesimal generator of $\left\{S_{K}^{\odot}(t)\right\}_{t \geq 0}$, where, by the previous discussion, $K^{\odot}$ is the part of $(A+B)^{*}$ in $X^{\odot}(K)$. Hence, the operators $(A+B)^{*}$ and $K^{\odot}$ are identical on $D\left(K^{\odot}\right)$ and the result then follows from [7, p.145].

\section{Test-Functions}

We now introduce a space of test-functions upon which, under appropriate conditions, the family of operators $\left\{S_{K}^{\odot}(t)\right\}_{t \geq 0}$ is equicontinuous and a corresponding family of restrictions is a semigroup of class $C_{0}$. We shall use the same space $Z$ as in [5], whose definition we recall for convenience. As usual, $\delta$ will denote the differential operator defined by

$$
(\delta \psi)(x)=x \psi^{\prime}(x) \quad(x>0)
$$

\section{Definition 3.1}

(i) The set $Z$ is defined by

$$
Z=\left\{\psi \in C^{\infty}\left(\mathbb{R}_{+}\right): \delta^{k} \psi \in Y \text { for } k=0,1,2, \ldots\right\}
$$

(ii) For each $k=0,1,2, \ldots$, define $\gamma_{k}$ and $\beta_{k}$ on $Z$ by

$$
\gamma_{k}(\psi)=\left\|x^{-1}\left(\delta^{k} \psi\right)\right\|_{\infty}=\left\|\delta^{k} \psi\right\|_{Y}, \quad \beta_{k}(\psi)=\left\|x^{-1}\left(x^{k} D^{k} \psi\right)\right\|_{\infty}=\left\|x^{k} D^{k} \psi\right\|_{Y}
$$

where $\|\cdot\|_{Y}$ is defined in (2.9).

It can be shown (see [5, Lemma 3.3]) that the families $\left\{\gamma_{k}\right\}_{k=0}^{\infty}$ and $\left\{\beta_{k}\right\}_{k=0}^{\infty}$ are countable multinorms on $Z$ (in the sense of [8, Section 1.6]) and generate equivalent topologies with respect to which $Z$ is a Fréchet space. 
Remark 3.2 Let $Z^{\prime}$ be the dual space of $Z$ equipped with the weak*-topology and let $\langle f, \psi\rangle$ denote the scalar onto which $\psi \in Z$ is mapped by $f \in Z^{\prime}$. Then the space $X$ can be imbedded in $Z^{\prime}$ via the mapping $\phi \rightarrow \tilde{\phi}$ where

$$
\langle\tilde{\phi}, \psi\rangle:=(\psi, \phi)=\int_{0}^{\infty} \psi(x) \phi(x) d x \quad(\phi \in X, \psi \in Z) .
$$

However, $Z^{\prime}$ also contains singular generalised functions such as $\delta(x-\ell)=\delta_{\ell}(\ell>0)$ defined by

$$
\left\langle\delta_{\ell}, \psi\right\rangle=\psi(\ell) \quad(\psi \in Z)
$$

The distributional ACPs studied below will have initial conditions belonging to $Z^{\prime}$.

We make the following assumption on the semigroup $\left\{S_{K}^{*}(t)\right\}_{t \geq 0}$.

(C.3) For all $t \geq 0$ and for each $i \in \mathbb{N}_{0}$, there exists $A_{i}>0$ independent of $t$ such that

$$
\beta_{i}\left(S_{K}^{*}(t) \psi\right) \leq A_{i} \sum_{r=0}^{i} \beta_{r}(\psi) \quad \forall \psi \in Z,
$$

where $\beta_{k}$ is defined in (3.3).

Theorem 3.3 If the semigroup $\left\{S_{K}^{*}(t)\right\}_{t \geq 0}$ satisfies (C.3), then $S_{K}^{*}(t) \in \mathcal{L}(Z)$ (the vector space of continuous linear operators on $Z$ ) for each $t \geq 0$, and $\left\{S_{K}^{*}(t)\right\}_{t \geq 0}$ is equicontinuous in $t$ on $Z$.

Proof Let $\left\{S_{K}^{*}(t)\right\}_{t \geq 0}$ satisfy (C.3). By [8, p.26], $S_{K}^{*}(t) \in \mathcal{L}(Z)$ for each $t \geq 0$. Moreover, for $\psi \in Z$ and $t \geq 0$,

$$
\beta_{i}\left(S_{K}^{*}(t) \psi\right) \leq A_{i} \sum_{r=0}^{i} \beta_{r}(\psi) \leq(i+1) A_{i} \max _{0 \leq r \leq i}\left[\beta_{r}(\psi)\right]=: \alpha_{i}(\psi) .
$$

By $\left[8\right.$, p.8], $\alpha_{i}$ is a continuous seminorm on $Z$. Hence, $\left\{S_{K}^{*}(t)\right\}_{t \geq 0}$ is equicontinuous in $t$ on $Z$; see $[9$, p.234].

Definition 3.4 We define the test-function semigroup dual of $X$ with respect to $\left\{S_{K}(t)\right\}_{t \geq 0}$, denoted by $Z(K)$, to be the linear subspace of $Z$ on which $S_{K}^{*}(t)$ is strongly continuous with respect to $t$, i.e.

$$
Z(K):=\left\{\psi \in Z: S_{K}^{*}(t) \psi \rightarrow \psi \text { in } Z \text { as } t \rightarrow 0^{+}\right\} .
$$

By the construction of $Z(K)$ and by the definition of $X^{\odot}(K)$,

$$
Z(K) \subset X^{\odot}(K)=\overline{D\left(K^{*}\right)}=\overline{D\left((A+B)^{*}\right)} .
$$


We now establish the basic properties of the space $Z(K)$ and analyse the behaviour of the operators $S_{K}^{*}(t)$ on $Z(K)$.

Theorem 3.5 The space $Z(K)$ is complete and so is a Fréchet space.

Proof Let $\left\{\psi_{n}\right\} \subset Z(K)$ be a Cauchy sequence. By the completeness of $Z$, there exists $\psi \in Z$ such that $\psi_{n} \rightarrow \psi$ in $Z$ as $n \rightarrow \infty$. Now, for each $i \in \mathbb{N}_{0}$,

$$
\begin{aligned}
& \beta_{i}\left(S_{K}^{*}(t) \psi-\psi\right) \\
\leq & \beta_{i}\left(S_{K}^{*}(t)\left(\psi-\psi_{n}\right)\right)+\beta_{i}\left(S_{K}^{*}(t) \psi_{n}-\psi_{n}\right)+\beta_{i}\left(\psi_{n}-\psi\right) \\
\leq & A_{i} \sum_{r=0}^{i} \beta_{r}\left(\psi-\psi_{n}\right)+\beta_{i}\left(S_{K}^{*}(t) \psi_{n}-\psi_{n}\right)+\beta_{i}\left(\psi_{n}-\psi\right)
\end{aligned}
$$

by (3.6). For any $\epsilon>0$, choose $n=N$ sufficiently large so that

$$
\beta_{r}\left(\psi-\psi_{N}\right)<\epsilon \quad \forall r=0,1,2, \ldots, i
$$

For this choice of $N$, choose $t=t_{0}$ sufficiently small so that

$$
\beta_{i}\left(S_{K}^{*}(t) \psi_{N}-\psi_{N}\right)<\epsilon \quad \text { for } 0<t \leq t_{0}
$$

Now, for this choice of $N$ and $t_{0}$,

$$
\beta_{i}\left(S_{K}^{*}(t) \psi-\psi\right) \leq\left[(i+1) A_{i}+2\right] \epsilon \quad \text { for } 0<t \leq t_{0} .
$$

Hence,

$$
\lim _{t \rightarrow 0^{+}} \beta_{i}\left(S_{K}^{*}(t) \psi-\psi\right)=0 .
$$

Therefore, $\psi \in Z(K)$ proving that $Z(K)$ is a complete countably multinormed space and hence a Fréchet space.

\section{Theorem 3.6}

(i) For each $t \geq 0$, the operator $S_{K}^{*}(t)$ leaves $Z(K)$ invariant, i.e. $S_{K}^{*}(t) \in \mathcal{L}(Z(K))$.

(ii) The family of operators $\left\{S_{K}^{*}(t)\right\}_{t \geq 0}$ is equicontinuous in $t$ on $Z(K)$.

\section{Proof}

(i) By Theorem 3.3, we know that $S_{K}^{*}(t) \in \mathcal{L}(Z(K), Z)$. Moreover, for any $t \geq 0$ and $\psi \in Z(K)$,

$$
\lim _{s \rightarrow 0^{+}}\left\{S_{K}^{*}(s) S_{K}^{*}(t) \psi-S_{K}^{*}(t) \psi\right\}=S_{K}^{*}(t) \lim _{s \rightarrow 0^{+}}\left[S_{K}^{*}(s) \psi-\psi\right]=0 .
$$

Hence $S_{K}^{*}(t)$ leaves $Z(K)$ invariant. 
(ii) This follows from Theorem 3.3 and (i).

Definition 3.7 For each $t \geq 0$, let

$$
S_{K}^{\times}(t):=S_{K}^{*}(t)_{\mid Z(K)}\left(=S_{K}^{\odot}(t)_{\mid Z(K)}\right)
$$

Theorem 3.8 The family $\left\{S_{K}^{\times}(t)\right\}_{t \geq 0}$ is an equicontinuous semigroup of class $C_{0}$ on $Z(K)$.

Proof By Theorem 3.6(i), $S_{K}^{\times}(t) \in \mathcal{L}(Z(K))$ for each $t \geq 0$. The semigroup properties of $\left\{S_{K}^{\times}(t)\right\}_{t \geq 0}$ are inherited from those of $\left\{S_{K}^{*}(t)\right\}_{t \geq 0}$ on $Y$, which contains $Z(K)$, with strong continuity being immediate from the definition of $Z(K)$. Equicontinuity follows from Theorem 3.6(ii).

Theorem 3.9 Let $K^{\times}$denote the infinitesimal generator of $\left\{S_{K}^{\times}(t)\right\}_{t \geq 0}$ on $Z(K)$. Then $K^{\times}$is the part of $(A+B)^{*}$ in $Z(K)$, i.e.

$$
D\left(K^{\times}\right):=\left\{\psi \in D\left((A+B)^{*}\right) \cap Z(K):(A+B)^{*} \psi \in Z(K)\right\}
$$

and

$$
K^{\times} \psi:=(A+B)^{*} \psi, \quad \psi \in D\left(K^{\times}\right) .
$$

Proof By Theorem 2.3, $\left\{S_{K}^{\odot}(t)\right\}_{t \geq 0}$ is contractive on $X^{\odot}(K)$. Further, $Z(K) \hookrightarrow X^{\odot}(K)$. Since $K^{\odot}$ is the infinitesimal generator of $\left\{S_{K}^{\odot}(t)\right\}_{t \geq 0}$, it follows that if $K_{\mid}$is the operator defined by

$$
D\left(K_{\mid}\right):=\left\{\psi \in D\left(K^{\odot}\right) \cap Z(K): K^{\odot} \psi \in Z(K)\right\},
$$

with

$$
K_{\mid} \psi:=K^{\odot} \psi, \quad \psi \in D\left(K_{\mid}\right),
$$

then $K_{\mid}$is the maximal operator on $Z(K)$ induced by $K^{\odot}$. Therefore,

$$
K^{\times}: Z(K) \supseteq D\left(K^{\times}\right) \rightarrow Z(K)
$$

is a restriction of $K_{\mid}$. Since $\left\{S_{K}^{\odot}(t)\right\}_{t \geq 0}$ is contractive, we know that

$$
R\left(\lambda, K^{\odot}\right):=\left(\lambda I-K^{\odot}\right)^{-1} \in B\left(X^{\odot}(K)\right) \text { for all } \lambda>0 \text {. }
$$

Also, from [10, p. 165], the equicontinuity of $\left\{S_{K}^{\times}(t)\right\}_{t \geq 0}$ on $Z(K)$ leads to

$$
R\left(\lambda, K^{\times}\right) \in \mathcal{L}(Z(K)) \quad \text { for all } \lambda>0,
$$


with

$$
R\left(\lambda, K^{\times}\right) \psi=\int_{0}^{\infty} e^{-\lambda t} S_{K}^{\times}(t) \psi d t=\int_{0}^{\infty} e^{-\lambda t} S_{K}^{\odot}(t) \psi d t=R\left(\lambda, K^{\odot}\right) \psi, \quad \text { for all } \psi \in Z(K) .
$$

For $\psi \in D\left(K_{\mid}\right)$,

$$
\psi=R\left(\lambda, K^{\odot}\right)\left(\lambda I-K^{\odot}\right) \psi=R\left(\lambda, K^{\odot}\right) \eta
$$

where $\eta:=\left(\lambda I-K^{\odot}\right) \psi \in Z(K)$. Now

$$
\psi=R\left(\lambda, K^{\odot}\right) \eta=R\left(\lambda, K^{\times}\right) \eta
$$

and hence $\psi \in D\left(K^{\times}\right)$. Therefore $K_{\mid}=K^{\times}$and the result follows since $K_{\mid}$is the part of $K^{\odot}$ in $Z(K)$ and, from $(2.14), K^{\odot}=(A+B)^{\odot}$.

Note that the previous theorem shows that the operators $K^{\times}$and $(A+B)^{*}$ are identical on $D\left(K^{\times}\right)$.

Corollary 3.10 The operator $S_{K}^{\times}(t)$ leaves $D\left(K^{\times}\right)$invariant and $\overline{D\left(K^{\times}\right)}=Z(K)$. Further, for each $t>0$ and $\psi \in D\left(K^{\times}\right)$,

$$
\frac{d}{d t} S_{K}^{\times}(t) \psi=S_{K}^{\times}(t) K^{\times} \psi=K^{\times} S_{K}^{\times}(t) \psi
$$

Proof Since $K^{\times}$is the infinitesimal generator of $\left\{S_{K}^{\times}(t)\right\}_{t \geq 0}$ on $Z(K)$, the results follow from [9, pp.237-240].

Theorem 3.11 The $A C P$

$$
\frac{d}{d t} u(t)=(A+B)^{*} u(t) \quad(t>0) ; \quad u(0)=u_{0},
$$

has a unique strongly continuously differentiable solution for all $u_{0} \in D\left(K^{\times}\right)$given by

$$
u(t)=S_{K}^{\times}(t) u_{0} \quad(t \geq 0) .
$$

Proof From Theorem 3.8, $\left\{S_{K}^{\times}(t)\right\}_{t \geq 0}$ is an equicontinuous semigroup of class $C_{0}$ on $Z(K)$, with infinitesimal generator $K^{\times}$, and so $u(t)=S_{K}^{\times}(t) u_{0}$ is the unique strongly continuously differentiable solution of the ACP

$$
\frac{d}{d t} u(t)=K^{\times} u(t) \quad(t>0) ; \quad u(0)=u_{0} \in D\left(K^{\times}\right) ;
$$

see [11, Theorem 2.2.37] for details. The result now follows immediately since $K^{\times}$and $(A+B)^{*}$ are identical on $D\left(K^{\times}\right)$. 


\section{Generalised Functions}

The space $Z^{\prime}(K)$ is the set of all continuous linear functionals on $Z(K)$ equipped with the weak*topology. By $[8, \mathrm{p} .21]$, and Theorem $3.5, Z^{\prime}(K)$ is sequentially complete. By standard results, the operator $\widetilde{S_{K}(t)}$ satisfying

$$
\left.\widetilde{\left\langle S_{K}(t)\right.} f, \psi\right\rangle=\left\langle f, S_{K}^{\times}(t) \psi\right\rangle \quad \text { for all } f \in Z^{\prime}(K), \psi \in Z(K),
$$

is the unique operator adjoint to $S_{K}^{\times}(t)$. Similarly, the unique operator adjoint to $K^{\times}$is the operator $\widetilde{K}$ satisfying

$$
\langle\widetilde{K} f, \psi\rangle=\left\langle f, K^{\times} \psi\right\rangle \quad \text { for all } f \in D(\widetilde{K}), \psi \in D\left(K^{\times}\right),
$$

where

$$
D(\widetilde{K}):=\left\{f \in Z^{\prime}(K): \exists g \in Z^{\prime}(K) \text { such that }\langle g, \psi\rangle=\left\langle f, K^{\times} \psi\right\rangle \forall \psi \in D\left(K^{\times}\right)\right\} .
$$

It follows from Theorem 3.9 that

$$
\langle\widetilde{K} f, \psi\rangle=\left\langle f,(A+B)^{*} \psi\right\rangle \quad \text { for all } f \in D(\widetilde{K}), \psi \in D\left(K^{\times}\right) .
$$

As indicated earlier, each function $\phi \in X$ can be associated with a functional $\widetilde{\phi} \in Z^{\prime}(K)$ that is defined by (3.4). Consequently, if $\phi \in D(A+B)=D(A)$ and $\eta=(A+B) \phi \in X$, then, for all $\psi \in Z(K)$,

$$
\langle\widetilde{\eta}, \psi\rangle=(\psi,(A+B) \phi)=\left((A+B)^{*} \psi, \phi\right)=\left\langle\widetilde{\phi},(A+B)^{*} \psi\right\rangle=\langle\widetilde{K} \widetilde{\phi}, \psi\rangle
$$

showing that we can interpret $\widetilde{K}$ as an extension of the operator $A+B$ to the space $Z^{\prime}(K)$.

Theorem 4.1 The family $\left\{\widetilde{S_{K}(t)}\right\}_{t \geq 0}$ is a weak*-continuous semigroup of linear operators on $Z^{\prime}(K)$ having $\widetilde{K}$ as its weak*-infinitesimal generator. Moreover, each $\widetilde{S_{K}(t)}$ leaves $D(\widetilde{K})$ invariant and, for each $t>0$ and $f \in D(\widetilde{K})$,

$$
\frac{d}{d t} \widetilde{S_{K}(t)} f=\widetilde{S_{K}(t)} \widetilde{K} f=\widetilde{K} \widetilde{S_{K}(t)} f .
$$

Proof The results follow from Theorem 3.8 and [12, pp.262-3].

Corollary 4.2 The ACP

$$
\frac{d}{d t} \widetilde{u}(t)=\widetilde{K} \widetilde{u}(t) \quad(t>0) ; \quad \widetilde{u}(0)=u_{0},
$$

has a unique weak*-continuously differentiable solution for all $u_{0} \in D(\widetilde{K})$ given by

$$
\widetilde{u}(t)=\widetilde{S_{K}(t)} u_{0}, \quad(t \geq 0) .
$$


Proof This can be deduced by applying analogous arguments to those used to establish [5, Corollary 3.13].

We construct the distributional extension $\widetilde{K}$ of $A+B$ with the intention of solving (4.4) when $u_{0}=\delta_{\ell}(=\delta(x-\ell))$. To obtain a unique strong solution $\widetilde{u}$ with respect to the topology on $Z^{\prime}(K)$, we require that $\delta_{\ell} \in D(\widetilde{K})$. In order to show this, we consider the multiplication operator $A$ defined in $X$ by (2.2). As $A$ is the infinitesimal generator of a $C_{0}$-semigroup $\left\{S_{A}(t)\right\}_{t \geq 0}$ of contractions on $X$, we can apply some of the theory of sun duals and the framework developed in the previous section to the operator $A$. Note that

$$
\left(S_{A}(t) \phi\right)(x)=e^{-a(x) t} \phi(x) \quad \text { for } t \geq 0, \phi \in X \text { and a.a. } x>0 .
$$

By [13, p.167], the operator $S_{A}^{*}(t)$ satisfying

$$
\left(S_{A}^{*}(t) \psi, \phi\right)=\left(\psi, S_{A}(t) \phi\right) \quad \text { for all } \phi \in X, \psi \in Y
$$

where $(\cdot, \cdot)$ is the duality defined by $(2.10)$, is the unique operator adjoint to $S_{A}(t)$. Similarly, the maximal operator $A^{*}$ satisfying

$$
\left(A^{*} \psi, \phi\right)=(\psi, A \phi) \quad \text { for all } \phi \in D(A), \psi \in D\left(A^{*}\right)
$$

is the unique operator adjoint to $A$. Routine calculations can be used to show that

$$
\begin{aligned}
& \left(A^{*} \psi\right)(x)=-a(x) \psi(x) \text { for a.a. } x>0 \text { and } \psi \in D\left(A^{*}\right), \\
& D\left(A^{*}\right)=\{\psi \in Y: a \psi \in Y\}, \\
& \left(S_{A}^{*}(t) \psi\right)(x)=e^{-a(x) t} \psi(x) \quad \text { for } t \geq 0, \psi \in Y \text { and a.a. } x>0 .
\end{aligned}
$$

The semigroup dual of $X$ with respect to $A$, denoted by $X^{\odot}(A)$, is given by

$$
X^{\odot}(A)=\left\{\psi \in Y: \lim _{t \rightarrow 0^{+}} S_{A}^{*}(t) \psi=\psi \text { in } Y\right\} .
$$

Similarly to Definition 2.2(ii), let

$$
S_{A}^{\odot}(t)=S_{A}^{*}(t)_{\mid X \odot(A)} .
$$

Then $\left\{S_{A}^{\odot}(t)\right\}_{t \geq 0}$ is a contractive $C_{0}$-semigroup on the Banach space $X^{\odot}(A)$ and

$$
X^{\odot}(A)=\overline{D\left(A^{*}\right)} .
$$

Also, if $A^{\odot}$ denotes the infinitesimal generator of $\left\{S_{A}^{\odot}(t)\right\}_{t \geq 0}$ on $X^{\odot}(A)$, then $A^{\odot}$ is the part of $A^{*}$ in $X^{\odot}(A)$.

We are now in a position to address the problem of whether $\delta_{\ell}$ lies in $D(\widetilde{K})$. 
Theorem 4.3 The functional $\delta_{\ell} \in D(\widetilde{K})$.

Proof Recall from (4.2) that

$$
D(\widetilde{K}):=\left\{f \in Z^{\prime}(K): \exists g \in Z^{\prime}(K) \text { such that }\langle g, \psi\rangle=\left\langle f, K^{\times} \psi\right\rangle \forall \psi \in D\left(K^{\times}\right)\right\},
$$

and let $\psi \in K^{\times}$. By definition,

$$
\left\langle\delta_{\ell}, K^{\times} \psi\right\rangle=\left(K^{\times} \psi\right)(\ell)=:\langle g, \psi\rangle .
$$

Now, by Theorems 3.9 and 2.4,

$$
K^{\times} \psi=(A+B)^{*} \psi=A^{*} \psi-A^{*} R^{*} \psi, \quad \forall \psi \in D\left(K^{\times}\right) .
$$

Therefore,

$$
\langle g, \psi\rangle=-a(\ell) \psi(\ell)+a(\ell)\left(R^{*} \psi\right)(\ell) .
$$

Now, it can be shown that $R^{*} \in \mathcal{L}(Z(K), Z)$ and therefore $R^{*} \psi \in Z$ for each $\psi \in Z(K)$. Hence $g$ is a well-defined linear functional on $Z(K)$. Also, if $\psi_{n} \rightarrow 0$ in $Z(K)$ then $R^{*} \psi_{n} \rightarrow 0$ in $Z$ and so $\left(R^{*} \psi_{n}\right)(x) \rightarrow 0$ pointwise for all $x>0$. Moreover,

$$
\psi_{n} \rightarrow 0 \text { in } Z(K) \Rightarrow x \psi_{n}(x) \rightarrow 0 \Rightarrow \psi_{n}(x) \rightarrow 0 \text { (pointwise for all } x>0 \text { ). }
$$

Hence, for each fixed $\ell>0$,

$$
\left\langle g, \psi_{n}\right\rangle=-a(\ell) \psi_{n}(\ell)+a(\ell)\left(R^{*} \psi_{n}\right)(\ell) \rightarrow 0 \text { as } n \rightarrow \infty .
$$

It follows that $g$ is continuous on $Z(K)$ and therefore, by definition, $\delta_{\ell} \in D(\widetilde{K})$.

Corollary 4.4 If $u_{0}=\delta_{\ell}$ in (4.4), then the unique weak*-continuously differentiable solution $\widetilde{u}$ of (4.4) for all $t \geq 0$ is given by $\widetilde{u}(t)=\widetilde{S_{K}(t)} \delta_{\ell}$.

Proof The result follows immediately from Corollary 4.2 and Theorem 4.3.

\section{A Particular Case}

We consider the particular case of (2.2) and (2.3) for which

$$
a(x)=x^{\alpha+1}, \quad r(x)=(\nu+2) x^{\nu}(x>0)
$$


where

$$
\alpha>-1, \quad-2<\nu \leq 0 .
$$

The condition $\nu \leq 0$ is found in [3] and is imposed here in view of the analysis which follows. The operator $A+B$ is therefore defined by

$$
\begin{aligned}
& (A \phi)(x)+(B \phi)(x)=-x^{\alpha+1} \phi(x)+(\nu+2) \int_{x}^{\infty}\left(\frac{x}{y}\right)^{\nu} y^{\alpha} \phi(y) d y \\
& D(A)=D(B):=\left\{\phi \in X: \int_{0}^{\infty} x^{\alpha+2}|\phi(x)| d x<\infty\right\}
\end{aligned}
$$

In contrast to the situation when $\alpha=-1$ studied in [5] where the corresponding operator $A+B$ is bounded on $X$, the natural domain, $D(A) \cap D(B)=D(A)$ of the expression in (5.3) is a proper subspace of $X$ and the resulting operator is not bounded.

Clearly conditions (C.1) and (C.2) are satisfied when $a$ and $r$ are given by (5.1) and therefore it only remains to verify that condition (C.3) also holds in this case. Fortunately, the semigroup generated by the closure $K$ of the operator $A+B$ defined via (5.3) and (5.4) is given explicitly by

$$
\begin{aligned}
& \left(S_{K}(t) \phi\right)(x) \quad:=e^{-x^{\alpha+1} t} \phi(x) \\
& \quad+\quad e^{-x^{\alpha+1} t}(\nu+2) t \int_{x}^{\infty}\left(\frac{x}{y}\right)^{\nu}{ }_{1} F_{1}\left(1-\gamma ; 2 ; t\left(x^{\alpha+1}-y^{\alpha+1}\right)\right) y^{\alpha} \phi(y) d y
\end{aligned}
$$

for all $\phi \in X, t \geq 0$ and a.a. $x>0$, where ${ }_{1} F_{1}$ denotes the confluent hypergeometric function and

$$
\gamma=\frac{\nu+2}{\alpha+1}
$$

see [14, Corollary 4.8]. A routine calculation involving Kummer's transformation then shows that

$$
\begin{aligned}
& \left(S_{K}^{*}(t) \psi\right)(x) \quad:=e^{-x^{\alpha+1} t} \psi(x) \\
& \quad+e^{-x^{\alpha+1} t}(\nu+2) t \int_{0}^{x}\left(\frac{y}{x}\right)^{\nu-\alpha}{ }_{1} F_{1}\left(1+\gamma ; 2 ; t\left(x^{\alpha+1}-y^{\alpha+1}\right)\right) y^{\alpha} \psi(y) d y
\end{aligned}
$$

for all $\psi \in Y, t \geq 0$ and a.a. $x>0$.

To simplify the calculations involved in verifying (C.3) for the semigroup defined via (5.7), it is convenient to use the operator $W$ defined on $Y$ by

$$
(W \psi)(x):=x^{(\nu-\alpha) /(\alpha+1)} \psi\left(x^{1 /(\alpha+1)}\right), \psi \in Y .
$$

This operator is an isometric isomorphism from $Y$ onto the Banach space $Y_{\gamma}$ given by

$$
Y_{\gamma}=\left\{\eta: \eta \text { is measurable on } \mathbb{R}_{+} \text {and }\|\eta\|_{Y_{\gamma}}<\infty\right\}
$$


where

$$
\|\eta\|_{Y_{\gamma}}:=\operatorname{ess} \sup _{x \in \mathbb{R}_{+}} x^{1-\gamma}|\eta(x)|=\left\|x^{1-\gamma} \eta(x)\right\|_{\infty} .
$$

Note that the conditions imposed on $\alpha$ and $\nu$ ensure that $\gamma>0$. Moreover, $Y_{2}=Y$. The inverse of $W$ is given by

$$
\left(W^{-1} \eta\right)(x):=x^{\alpha-\nu} \eta\left(x^{\alpha+1}\right), \eta \in Y_{\gamma}
$$

If we now define

$$
T_{\gamma}(t) \eta:=W S_{K}^{*}(t) W^{-1} \eta, \eta \in Y_{\gamma}
$$

then it follows that $\left\{T_{\gamma}\right\}_{t \geq 0}$ is a semigroup of linear operators on $Y_{\gamma}$, and, from (5.7), (5.8) and (5.11),

$$
\left(T_{\gamma}(t) \eta\right)(x):=e^{-x t} \eta(x)+\gamma t e^{-x t} \int_{0}^{x}{ }_{1} F_{1}(1+\gamma ; 2 ; t(x-y)) \eta(y) d y .
$$

The advantage of working with (5.12), instead of (5.7), is that only one parameter is involved instead of two, and also awkward powers of $x$ and $y$ have been eliminated.

To make further progress, we must verify that the semigroup $\left\{T_{\gamma}(t)\right\}_{t \geq 0}$ satisfies an inequality akin to (3.6) on an appropriately defined space of test-functions $Z_{\gamma}$. In the following definition, $\delta$ will again denote the differential operator $x d / d x$.

\section{Definition 5.1}

(i) The set $Z_{\gamma}$ is defined by

$$
Z_{\gamma}=\left\{\eta \in C^{\infty}\left(\mathbb{R}_{+}\right): \delta^{k} \eta \in Y_{\gamma} \text { for } k=0,1,2, \ldots\right\}
$$

(ii) For each $k=0,1,2, \ldots$, define $\alpha_{k}^{\gamma}$ and $\beta_{k}^{\gamma}$ on $Z_{\gamma}$ by

$$
\alpha_{k}^{\gamma}(\eta)=\left\|x^{1-\gamma}\left(\delta^{k} \eta\right)\right\|_{\infty}=\left\|\delta^{k} \eta\right\|_{Y_{\gamma}}, \beta_{k}^{\gamma}(\eta)=\left\|x^{1-\gamma}\left(x^{k} D^{k} \eta\right)\right\|_{\infty}=\left\|x^{k} D^{k} \eta\right\|_{Y_{\gamma}} .
$$

It can be shown that the families $\left\{\alpha_{k}^{\gamma}\right\}_{k=0}^{\infty}$ and $\left\{\beta_{k}^{\gamma}\right\}_{k=0}^{\infty}$ are countable multinorms on $Z_{\gamma}$ and generate equivalent topologies with respect to which $Z_{\gamma}$ is a Fréchet space. Note also that $Z_{2}=Z$.

Lemma 5.2 Under the conditions (5.2), $W$ is homeomorphism from $Z$ onto $Z_{\gamma}$.

Proof We have already observed that $W$ is an isometric isomorphism from $Y$ onto $Y_{\gamma}$. We now observe that, for each $k \in \mathbb{N}$,

$$
\delta^{k}(W \psi)=W\left[(\alpha+1)^{-1}(\delta-(\alpha-\nu)]^{k} \psi, \forall \psi \in Z,\right.
$$


and

$$
\delta^{k}\left(W^{-1} \eta\right)=W^{-1}[(\alpha+1) \delta+(\alpha-\nu)]^{k} \eta, \quad \forall \eta \in Z_{\gamma} .
$$

The result then follows from Definitions 3.1 and 5.1.

Lemma 5.3 For each $n \in \mathbb{N}_{0}$ and for $\eta \in Z_{\gamma}$,

$$
\beta_{n}^{\gamma}\left(T_{\gamma}(t) \eta\right) \leq \sum_{r=0}^{n} d_{r} \beta_{r}^{\gamma}(\eta),
$$

where $d_{r}(r=0,1, \ldots, n)$ are constants independent of $t$.

Proof Differentiating the second term in (5.12) gives

$$
\begin{aligned}
& \frac{d}{d x}\left(t e^{-x t} \int_{0}^{x}{ }_{1} F_{1}(1+\gamma ; 2 ; t(x-y)) \eta(y) d y\right) \\
= & \frac{d}{d x}\left(t \int_{0}^{x}{ }_{1} F_{1}(1-\gamma ; 2 ; t(y-x)) e^{-y t} \eta(y) d y\right) \\
= & t e^{-x t} \eta(x)-\frac{t^{2}}{2}(1-\gamma) \int_{0}^{x}{ }_{1} F_{1}(2-\gamma ; 3 ; t(y-x)) e^{-y t} \eta(y) d y
\end{aligned}
$$

since, by [15, p.254(8)],

$$
\frac{d}{d x}{ }_{1} F_{1}(a ; b ; x)=\frac{a}{b}{ }_{1} F_{1}(a+1 ; b+1 ; x) .
$$

By induction, for appropriate constants $a_{r}, b_{r}$ and $c_{r}$ depending on $\gamma$,

$$
\begin{aligned}
& \frac{d^{n}}{d x^{n}}\left(t e^{-x t} \int_{0}^{x}{ }_{1} F_{1}(1+\gamma ; 2 ; t(x-y)) \eta(y) d y\right) \\
= & \sum_{r=0}^{n-1} a_{r}(\gamma) t^{n-r} e^{-x t} \eta^{(r)}(x)+b_{n}(\gamma) t^{n+1} \int_{0}^{x}{ }_{1} F_{1}(1-\gamma+n ; 2+n ; t(y-x)) e^{-y t} \eta(y) d y \\
\Rightarrow & x^{n} \frac{d^{n}}{d x^{n}}\left(t e^{-x t} \int_{0}^{x}{ }_{1} F_{1}(1+\gamma ; 2 ; t(x-y)) \eta(y) d y\right) \\
= & \sum_{r=0}^{n-1} a_{r}(\gamma)(x t)^{n-r} e^{-x t} x^{r} \eta^{(r)}(x)+c_{n}(\gamma)(x t)^{n} t e^{-x t} \int_{0}^{x}{ }_{1} F_{1}(1+\gamma ; 2+n ; t(x-y)) \eta(y) d y .
\end{aligned}
$$

Now $y^{n-r} e^{-y}$ is bounded for $0 \leq r \leq n$ and $y \geq 0$. Hence

$$
\left\|(x t)^{n-r} e^{-x t} x^{r} \eta^{(r)}(x)\right\|_{Y_{\gamma}} \leq M_{r}\left\|x^{r} \eta^{(r)}(x)\right\|_{Y_{\gamma}}
$$

for some constant $M_{r}$ which is independent of $t \geq 0$. Also 


$$
\begin{aligned}
& \left|(x t)^{n} t e^{-x t} \int_{0}^{x}{ }_{1} F_{1}(1+\gamma ; 2+n ; t(x-y)) \eta(y) d y\right| \\
= & \left|(x t)^{n} t e^{-x t} \int_{0}^{x} y^{\gamma-1}{ }_{1} F_{1}(1+\gamma ; 2+n ; t(x-y)) y^{1-\gamma} \eta(y) d y\right| \\
\leq & (x t)^{n} t e^{-x t}\|\eta\|_{Y_{\gamma}} \int_{0}^{1}[x(1-u)]^{\gamma-1}{ }_{1} F_{1}(1+\gamma ; 2+n ; t x u) x d u \\
= & (x t)^{n} t e^{-x t}\|\eta\|_{Y_{\gamma}} x^{\gamma} \frac{\Gamma(\gamma)}{\Gamma(\gamma+1)}{ }_{2} F_{2}(1,1+\gamma ; 1+\gamma, 2+n ; t x) \quad \text { by [16, p.807(12)]) } \\
= & (x t)^{n} t e^{-x t}\|\eta\|_{Y_{\gamma}} x^{\gamma} \frac{\Gamma(\gamma)}{\Gamma(\gamma+1)}{ }_{1} F_{1}(1 ; 2+n ; t x) \\
\leq & b_{\gamma}(x t)^{n} t e^{-x t}\|\eta\|_{Y_{\gamma}} x^{\gamma} e^{x t}(x t)^{-1-n} \quad \text { by }[15, \mathrm{p} .278(3)] \\
= & x^{\gamma-1} b_{\gamma}\|\eta\|_{Y_{\gamma}} \quad\left(\text { for some constant } b_{\gamma}\right) .
\end{aligned}
$$

Hence,

$$
\left\|(x t)^{n} t e^{-x t} \int_{0}^{x}{ }_{1} F_{1}(1+\gamma ; 2+n ; t(x-y)) \eta(y) d y\right\|_{Y_{\gamma}} \leq b_{\gamma}\|\eta\|_{Y_{\gamma}} .
$$

Using (5.18) and (5.19) and incorporating the first term in (5.12) gives (5.17).

We can now establish that $\left\{S_{K}^{*}(t)\right\}_{t \geq 0}$ satisfies condition (C.3).

Theorem 5.4 For each $n \in \mathbb{N}_{0}$, there exists a constant $A_{n}>0$, independent of $t \geq 0$ such that

$$
\beta_{n}\left(S_{K}^{*}(t) \gamma(t) \psi\right) \leq A_{n} \sum_{r=0}^{n} \beta_{r}(\psi), \quad \forall \psi \in Z .
$$

Proof This follows immediately from Lemmas 5.3 and 5.2 on using the definitions of the seminorms in (3.3) and (5.14). Notice in particular that the expressions on the right-hand sides of (5.15) and (5.16) involve polynomials in $\delta$ whose coefficients are independent of $t \geq 0$.

If we now take $\widetilde{K}$ to be the distributional extension (defined via (4.3)) of the specific operator $A+B$ given by (5.3) and (5.4), then Corollary 4.2 shows that the corresponding ACP

$$
\frac{d}{d t} \widetilde{u}(t)=\widetilde{K} \widetilde{u}(t) \quad(t>0) ; \quad \widetilde{u}(0)=u_{0}
$$

has a unique weak*-continuously differentiable solution $\widetilde{u}$ for all $u_{0} \in D(\widetilde{K})$. Moreover,

$$
\widetilde{u}(t)=\widetilde{S_{K}(t)} u_{0}, \quad(t \geq 0),
$$

where $\widetilde{S_{K}(t)}$ is defined by (4.1).

By Theorem 4.3, the functional $\delta_{\ell} \in D(\widetilde{K})$. Hence the unique weak*-continuously differentiable solution $\widetilde{u}$ of $(5.21)$ for all $t \geq 0$ when $u_{0}=\delta_{\ell}$ is given by $\widetilde{u}(t)=\widetilde{S_{K}(t)} \delta_{\ell}$. 
Definition 5.5 For each $-2<\nu \leq 0, \alpha>-1, \ell>0$ and $t>0$, the function $k_{\nu, \alpha, \ell, t}$ is defined by

$$
k_{\nu, \alpha, \ell, t}(x):=(\nu+2) t \ell^{\alpha-\nu} e^{-\ell^{\alpha+1} t} H(\ell-x) x^{\nu}{ }_{1} F_{1}\left(1+\gamma ; 2 ; t \ell^{\alpha+1}-t x^{\alpha+1}\right), x>0,
$$

where $H$ denotes the Heaviside function and $\gamma$ is defined by (5.6).

Lemma 5.6 The function $k_{\nu, \alpha, \ell, t} \in X$ and satisfies

$$
\left\|k_{\nu, \alpha, \ell, t}\right\|_{X} \leq \ell\left\|K_{1}\right\|_{\infty}, \text { where } K_{1}(x)=x e^{-x}{ }_{1} F_{1}(1 ; 2 ; x) .
$$

Proof We have

$$
\begin{aligned}
& \left\|k_{\nu, \alpha, \ell, t}\right\|_{X} \\
= & (\nu+2) t \ell^{\alpha-\nu} e^{-\ell^{\alpha+1} t} \int_{0}^{\ell} x^{\nu+1}{ }_{1} F_{1}\left(1+\gamma ; 2 ; t \ell^{\alpha+1}-t x^{\alpha+1}\right) d x \\
= & (\nu+2) t \ell^{\alpha+1} e^{-\ell^{\alpha+1} t} \int_{0}^{\ell}(x / \ell)^{\nu+1}{ }_{1} F_{1}\left(1+\gamma ; 2 ; t \ell^{\alpha+1}-t x^{\alpha+1}\right) d x \\
= & \gamma t \ell^{\alpha+2} e^{-\ell^{\alpha+1}} t \int_{0}^{1}(1-y)^{\gamma-1}{ }_{1} F_{1}\left(1+\gamma ; 2 ; t \ell^{\alpha+1} y\right) d y \\
= & \gamma t \ell^{\alpha+2} e^{-\ell^{\alpha+1} t} \frac{\Gamma(\gamma)}{\Gamma(\gamma+1)}{ }_{2} F_{2}\left(1,1+\gamma ; 1+\gamma, 2 ; t \ell^{\alpha+1}\right) \quad(\text { by }[16, p .807(12)]) \\
= & t \ell^{\alpha+2} e^{-\ell^{\alpha+1} t}{ }_{1} F_{1}\left(1 ; 2 ; t \ell^{\alpha+1}\right)=\ell K_{1}\left(t \ell^{\alpha+1}\right) \quad(\text { by }[15, p .278(3)]),
\end{aligned}
$$

where the function $K_{1}$ is defined in (5.23). Since $K_{1} \in Y$, the result follows.

Theorem 5.7 Let $k_{\nu, \alpha, \ell, t}$ be defined by (5.22). If $-2<\nu \leq 0$ and $\alpha>-1$, then

$$
\widetilde{S_{K}(t)} \delta_{\ell}=e^{-\ell^{\alpha+1} t} \delta_{\ell}+\widetilde{k_{\nu, \alpha, \ell, t}}
$$

for all $t \geq 0$. Hence the solution $\widetilde{u}$ to (5.21) when $u_{0}=\delta_{\ell}$ is given by

$$
\widetilde{u}(t)=e^{-\ell^{\alpha+1} t} \delta_{\ell}+\widetilde{k_{\nu, \alpha, \ell, t}} \text {. }
$$

Proof This follows on evaluating $\left\langle\widetilde{S_{K}(t)} \delta_{\ell}, \psi\right\rangle$ for $\psi \in Z(K)$. The calculation is routine and details are omitted.

The calculations required to prove the previous theorem establish that

$$
\begin{aligned}
\widetilde{S_{K}(t)} \delta_{\ell} & =e^{-\ell^{\alpha+1} t} \delta_{\ell} \\
& +\left[(\nu+2) t \ell^{\alpha-\nu} e^{-x^{\alpha+1} t} H\left(\widehat{\ell-x) x^{\nu}}{ }_{1} F_{1}\left(1-\gamma ; 2 ; t x^{\alpha+1}-t \ell^{\alpha+1}\right)\right] .\right.
\end{aligned}
$$

When $\nu=0$,

$$
\begin{aligned}
\widetilde{S_{K}(t)} \delta_{\ell} & =e^{-\ell^{\alpha+1} t} \delta_{\ell} \\
& +\left[2 t \ell^{\alpha} e^{-x^{\alpha+1} t} H \widetilde{(\ell-x)}{ }_{1} F_{1}\left(\frac{\alpha-1}{\alpha+1} ; 2 ; t x^{\alpha+1}-t \ell^{\alpha+1}\right)\right] .
\end{aligned}
$$


Formula (5.25) coincides with the solution obtained in $[2$, p.2515(3)]. Once again, the advantage of our method is that we have obtained (5.25) using rigorous mathematical techniques. Moreover, our solution is valid for $-2<\nu \leq 0$ and so our result can be regarded as being more general than that given in $[2]$.

\section{References}

[1] R. M. Ziff and E. D. McGrady. The kinetics of cluster fragmentation and depolymerisation. J. Phys. A : Math. Gen. 1985; 18:3027-3037.

[2] R. M. Ziff and E. D. McGrady. Kinetics of polymer degradation. Macromolecules 1986; 19: $2513-2519$.

[3] E. D. McGrady and R M. Ziff. "Shattering" transition in fragmentation. Phys. Rev. Lett. 1987; 58: $892-895$.

[4] J. Banasiak and L. Arlotti. Positive Perturbations of Semigroups with Applications. Springer: London, 2006.

[5] W. Lamb, A. C. McBride and G. C. McGuinness. Fragmentation arising from a distributional initial condition. Math. Methods Appl. Sci. 2010; 33: 1183-1191.

[6] J. Banasiak. Shattering and non-uniqueness in fragmentation models - an analytic approach. Physica D 2006; 222: 63-72.

[7] K.-J. Engel and R. Nagel. One-parameter Semigroups for Linear Evolution Equations. Springer: New York, 2000.

[8] A. H. Zemanian. Generalized Integral Transformations (2nd. ed.). Dover: New York, 1987.

[9] K. Yosida. Functional Analysis (Sixth Edition). Springer-Verlag: Berlin, 1980.

[10] I. Miyadera. Semi-groups of operators in Fréchet space and applications to partial differential equations. Tohoku Math. J. 1959; 11: 162-183.

[11] G. C. McGuinness. A Distributional Approach to Fragmentation Equations. Ph.D. Thesis, University of Strathclyde, Glasgow, 2006.

[12] T. Kōmura. Semigroups of operators in locally convex spaces. J. Functional Anal. 1968; 2: 258-296. 
[13] T. Kato. Perturbation Theory for Linear Operators (Second Edition). Springer-Verlag: Berlin, 1976.

[14] P. N. Blair, W. Lamb and I. W. Stewart. Coagulation and fragmentation with discrete mass loss, J. Math. Anal. Appl. 2007; 329: 1285-1302.

[15] A. Erdélyi et al. Higher Transcendental Functions Vol. 1, McGraw-Hill, New York, 1953.

[16] I. S. Gradshteyn and I. M. Ryzhik. Tables of Integrals, Series and Products (Sixth Edition). Academic Press: San Diego, 2000. 